Objective: The aim of this study was to compare the rehabilitative outcomes of 2 common approaches in total hip arthroplasties, in the subacute setting.

Design: We obtained a comprehensive list of patients admitted for subacute rehabilitation of total hip arthroplasties between July 2009 and February 2011. Based on the surgical approach documented in electronic medical records, we then separated the patients into the anterior approach group and the posterior approach group, and then compared averages: length of stay, admission Functional Independence Measure (FIM) scores, discharge FIM scores, and FIM gain and dispositions.

Setting: A subacute rehabilitation unit.

Participants: Admissions to subacute rehabilitation from July 2009 to February 2011 after total hip arthroplasties.

Interventions: Not applicable.

Main Outcome Measures: Length of stay, admission FIM score, discharge FIM score, and FIM gain and disposition after rehabilitation.

Results: During our time frame, 60 patients were admitted to the subacute rehabilitation unit after total hip arthroplasties. Of the 60, only 10 underwent anterior approaches (16.6\%). Admission FIM scores were found to be significantly higher in the anterior approach group $(P=.028)$ than in the posterior approach group. Comparison of the two groups' length-of-stays shows the anterior approach group tended to be shorter than the posterior approach group $(P=.106)$. However, no significant difference was found between the 2 groups in discharge FIM scores $(P=.164)$ and FIM gains $(P=.364)$. Discharge FIM scores tended to be higher in the anterior approach group, whereas FIM gains tended to be higher in the posterior approach group.

Conclusions: Patients undergoing postoperative subacute rehabilitation for anterior total hip arthroplasties have significantly higher admission FIM scores compared with patients with posterior approach total hip arthroplasties. Anterior approach total hip arthroplasties also tend to have shorter lengths-of-stay and higher discharge FIM scores then posterior total hip arthroplasties. Significantly higher admission FIM scores support the idea that anterior hip precautions are less restrictive of many activities of daily living, when compared with posterior precautions.

\section{Poster 156}

\section{Musculoskeletal Injuries in Dancers.}

Devon Shuchman, MD, MS (University of Michigan, Ann Arbor, MI, United States); Joseph E. Hornyak, MD, PhD, Michael J. Louwers, MD.

Disclosures: D. Shuchman, none.

Objective: Our objective was to determine frequency, type, and severity of musculoskeletal injuries in dancers. We aimed to identify barriers to medical care for dancers and categories of dance with increased risk of acute injury and chronic pain.

Design: This is a cross-sectional survey, anonymous and Web based. There is no direct contact with subjects. The study was approved by the institutional review board.

Setting: The subjects were recruited from U.S. dancer's alliances, organizations, and performance venues.

Participants: Inclusion criteria are amateur or professional dancers at least 18 years of age. Subjects self-identify as modern, classical, or alternative dancers. Subclassification of dance also was identified as line, jazz, tap, hip-hop, ballet, ballroom, Latin, pole, exotic, or other.

Interventions: A Web-based survey is used to query targeted populations of both amateur and professional dancers.

Main Outcome Measures: The survey is designed to obtain data on the frequency, type, and severity of musculoskeletal injuries in dancers. This survey assesses functional limitations placed on the dancers because of these injuries and also identifies the medical attention sought or received.

Results: The current number of 88 subjects: 68 classical, 11 modern, 3 alternative; $55 \%$ between ages 18 and 22 years old, with $95 \%$ women; $41 \%$ report having obtained at least a 4 -year degree. The mean height was $5 \mathrm{ft} 5 \mathrm{in}$. ( $4 \mathrm{ft} 6 \mathrm{in}$. to $6 \mathrm{ft} 1 \mathrm{in}$.), mean weight of $134.93 \mathrm{lb}(90-210 \mathrm{lb})$ and mean years of experience, $7.4 ; 73 \%$ of those with chronic or recurrent back pain are heel wearers (2-in. heels or taller); $89 \%$ sought medical attention for an injury in the past 6 months; 93\% denied that pain interferes with work. Data analysis is ongoing.

Conclusions: Chronic pain prevalence is higher than previously reported in all dancers. Heel height is a significant factor in injury prevalence. The incidence of acute injury is equal to or greater than that reported in the current literature. A significant barrier to access medical care is insurance coverage. Dancers of all ages and categories have positive health perceptions and attitudes despite a high prevalence of chronic pain. This study has the ability to identify modifiable risks of injury in specific dance populations, to propose injury prevention strategies, increase practitioner awareness of most prevalent injuries in dancers, and allow for efficient and effective care for patients.

\section{Poster 157}

Relationship Between Psychosocial Factors and Disability in Patients With Chronic Low Back Pain. Tonatiuh Avila (National Institute of Rehabilitation, Mexico City, Mexico); Eva Cruz, Silvia Porras, Monica Sandoval, Family Physician.

Disclosures: T. Avila, none.

Objective: To evaluate the relationship between psychosocial factors and disability in patients with chronic low back pain.

Design: Cross-sectional study.

Setting: Outpatient rehabilitation center.

Participants: 67 subjects with mechanical low back pain or spondyloarthrosis and a history of at least 10 sessions of physical therapy; previous spine surgery, spondylolisthesis, rheumatic diseases, bone infections, and malignancies were exclusion criteria.

Interventions: Not applicable.

Main Outcome Measures: Self-reported disability, pain intensity, fear of movement, and depression measured with the Roland Morris Questionnaire, visual analog scale, Tampa Scale for Kinesiophobia, and Beck Depression Inventory, respectively; body mass index and the presence of yellow flags were also evaluated. Differences in self-reported disability related with the presence of yellow flags and overweight or obesity were analyzed by the t-test; the coefficient of Pearson was used to analyze the correlation between psychosocial factors and self-reported disability.

Results: Self-reported disability was significantly greater in patients with previous change of work activity as a result of lumbar pain $(P=.02)$, financial problems $(P<.01)$, and overweight or obesity $(P=.02)$. Weak but statistically significant correlations between 